\title{
VISUALIZATION OF BIG GEODATA: AN EXPERIMENT WITH DINSAR DEFORMATION TIME SERIES
}

\author{
Candan Eylül Kilsedar ${ }^{1}$, Luca Frigerio ${ }^{2}$, Manuela Bonano ${ }^{2,3}$, Gloria Bordogna ${ }^{2}$, Paola Carrara ${ }^{2}$, Pasquale Imperatore ${ }^{2}$, Riccardo \\ Lanari $^{2}$, Mariarosaria Manzo ${ }^{2}$, Antonio Pepe ${ }^{2}$, Maria Antonia Brovelli ${ }^{1}$ \\ ${ }^{1}$ Politecnico di Milano, Department of Civil and Environmental Engineering, Piazza Leonardo da Vinci 32, 20133 Milan, Italy - \\ (candaneylul.kilsedar, maria.brovelli)@polimi.it \\ ${ }^{2}$ CNR Institute for the Electromagnetic Sensing of the Environment (IREA), Diocleziano 328, 80124 Naples, Italy - (frigerio.1, \\ bonano.m, bordogna.g, carrara.p, imperatore.p, lanari.r, manzo.mr, pepe.a)@irea.cnr.it \\ ${ }^{3}$ CNR Institute of Methodologies for Environmental Analysis (IMAA), C. da S. Loya, 85050 Tito Scalo, Potenza, Italy - \\ manuela.bonano@imaa.cnr.it
}

Commission IV, WG IV/4

KEY WORDS: Big Geodata, Interoperability, DInSAR Deformation Time Series, Geovisualization, Visual Exploration, Cartographic Animation, Virtual Globes

\begin{abstract}
:
This paper describes an experiment carried out within the project "URBAN GEOmatics for Bulk data Generation, Data Assessment and Technology Awareness (URBAN GEO BIG DATA)" on novel technological solutions for encouraging the use of time series of big geodata by scientists, public administrators, and the citizens of urban areas. In this experiment, we focus on fostering the use of ground deformation time series derived through the Differential Synthetic Aperture Radar Interferometry (DInSAR) measurements in urban areas (i.e., Naples, Milan, and Turin) through visualization. Two visualization applications are developed within the project. First, mean deformation velocity maps are visualized; second, the cumulative deformation time series is animated to enable the comprehension of deformation of eighteen years at a glance. The experiment allows experts and non-experts to discover spatio-temporal patterns of deformation. The paper focuses on the characteristics and implementation details of this experiment. Both visualizations are overlaid on a three-dimensional map, i.e., a virtual globe. We use open standards and free and open source software (FOSS) for Web to enable interoperability, replicability, and reusability.
\end{abstract}

\section{INTRODUCTION}

Big GeoData (BGD) constitute a challenge for monitoring and assessing the status of and changes in both the natural and the built environment where most people live. Nevertheless, to convert BGD into added value, we need to fill the gap between the current form in which BGD is represented, which conveys information understandable to experts, particularly scientists, and the needs of public administrators and citizens who could exploit the information derived from BGD if adequately summarized and visualized. To this end, new methods are needed for the discovery of the relevant geodata among huge repositories, the assessment of the geodata quality, and finally, the synthesis of BGD to provide non-experts with consistent and comprehensible information to possibly retrieve hidden knowledge.

Within the project "URBAN GEOmatics for Bulk data Generation, Data Assessment and Technology Awareness (URBAN GEO BIG DATA)" we have experimented with the definition and application of novel technological solutions for encouraging the use of BGD by scientists, public administrators, and the citizens of urban areas. Specifically, the project aims to improve the knowledge of urban areas by exploiting the vast amount of Earth Observation (EO) data sources for ground deformation, soil consumption, land use and land cover available for long-term monitoring, Internet of Things (IoT) data on mobility, and three-dimensional city visualization, which currently involves only buildings. A key aspect concerns the definition and implementation of novel methods for geodata dissemination through the application and extension of standard interoperable sharing protocols.

In this paper, we focus on the realization of ground deformation time series derived through the Differential Synthetic Aperture Radar Interferometry (DInSAR) measurements in urban areas (i.e., Naples, Milan, and Turin). In particular, the Small BAseline Subset (SBAS) technique is applied to generate DInSAR BGD deformation time series. The mean deformation velocity maps and time series data can be retrieved by making Open Geospatial Consortium (OGC) Web Map Service (WMS) and Web Feature Service (WFS) requests; however, it is hard to interpret the data by non-experts.

Besides the mean deformation velocity maps, we defined and implemented an automatic mechanism aimed at generating a temporal animation of the BGD time series of deformation, composed of maps generated with a reduced spatial and temporal resolution for the analyzed areas. They can be helpful for non-experts to visually identify at a glance the areas subject to deformation, without spending much time analyzing the single deformation time series. To follow the time evolution of the deformation, we selected merely one single measurement per year. This is a method for helping non-experts in identifying areas with large deformations. The paper focuses on this aspect describing its characteristics and implementation details. Specifically, Section 2 describes the related works. Section 3 outlines the main steps of the algorithm for the DInSAR time series generation. Section 4 describes the automatic procedure for DInSAR time series interoperable sharing. Section 5 
describes the CesiumJS application for DInSAR time series visualization. The final section summarizes the main results and future work.

\section{BASIC NOTIONS OF TEMPORAL GEOVISUALIZATION}

The focus of this paper is the methods we use to generate the BGD time series and their visualization. Visualization refers to the process in which a series of transformations converts raw data into images to convey information understandable by humans for a given purpose. Thus, designing a visualization modality that takes into account both humans' perception and knowledge is fundamental.

Scientific visualization is concerned with the visualization of scientific phenomena mainly offered to enable scientists to understand and possibly discover something from their data. Thus suitable visualization modalities are those favoring exploratory data analysis and synthesis. Computer graphics and computer animation provide great help for effective scientific visualization of both bi-dimensional and three-dimensional data.

Geovisualization is a form of information visualization in which principles from cartography, geographic information systems (GISs), exploratory data analysis, and information visualization are integrated into the development and assessment of visual methods that facilitate the exploration, analysis, synthesis, and presentation of georeferenced information (MacEachren et al., 2001). GISs and Web mapping tools and services implement geovisualization methods based on cartographic rules.

When the visualization of BGD time series targets non-experts, the described spatio-temporal phenomena can be perceived more effectively by temporal animated geo-mapping. Andrienko et al. (2001) assert that "in order to be able to think about data, the mind needs to perceive the data." In that sense, they consider that "one picture is worth much more than a collection of numbers." Furthermore, Shneiderman (1996) states that any visual exploration task should begin with an overview, or overall representation of the data and Ogao and Kraak (2002) add that temporal animated maps offer "the opportunity to deal with real-world processes as a whole." Peuquet (1994) developed a conceptual framework for the integration of time into geovisualization based on the concepts "where - when what" in which time is a dimension itself. According to Peuquet, geographic phenomena can be described from three different perspectives:

- when + where $\rightarrow$ what: it describes the phenomenon (what) that occurred at a given location/s (where) at a given time/s (when).

- when + what $\rightarrow$ where: it describes the location/s (where) affected by a given phenomenon (what) at a given time/s (when).

- where + what $\rightarrow$ when: it describes the time/s (when) that a given phenomenon (what) occurred at a given location $/ \mathrm{s}$ (where).

Each perspective is suitable for a different exploratory task. For instance, to focus on when, the where + what perspective is the most suitable to explore the evolution of the overall phenomenon over time. To this end, changes in a time interval can be represented differently: as existential changes, i.e., appearances and disappearances; as changes of spatial properties (location, shape, size, orientation, altitude, gradient, etc.); as changes of thematic properties represented by values of attributes. In this perspective, temporal animated geo-mapping is the most common representation in which the animation is performed by a sequence of static frames, the visual content of which, when shown in rapid succession, begins moving in a fluid motion (Harrower and Fabrikant, 2008). Temporal animated geo-mapping allows detecting spatio-temporal patterns. For instance, it can ease the identification of increasing/decreasing/periodic characteristics of ground deformation, soil consumption, traffic, etc. In the following of this paper, an experiment of temporal geovisualization of DInSAR measurements in urban areas will be described based on the implementation of standard interoperable Web mapping services.

\section{DINSAR TIME SERIES GENERATION}

DInSAR currently represents a suitable and well-established technique for the mapping and continuous monitoring of areas on Earth that are subject to ground deformation (Massonnet et al., 1998). In particular, DInSAR can measure the deformation occurred on the ground between two (or more) flight passes of the same radar sensor over an Area of Interest (AOI) by extracting the phase difference between the Synthetic Aperture Radar (SAR) images taken from slightly different orbital positions and at different acquisition times. Originally developed to analyze single deformation episodes (Peltzer et al., 1995), DInSAR has been extended over the years to investigate the temporal evolution of the surface deformation through the generation of long-term displacement time series. In this context, several approaches have been developed (Ferretti et al., 2001; Kampes, 2006; Berardino et al., 2002; Mora et al., 2003), which can be globally categorized in the two main classes referred to as the Permanent Scatterers (PS) (Ferretti et al., 2001; Kampes, 2006) and the Small Baseline (SB) (Berardino et al., 2002; Mora et al., 2003) methods.

The PS technique at the scale of resolution of single pixels is based on the discrimination of the deformation signals related to high-coherent, pointwise structures on the ground (i.e., the so-called permanent scatterers), which preserve the deformation information even when using large-baseline interferograms. On the contrary, the SB technique consists of methods based on the selection of a proper set of SB interferograms, which are used for the study of the deformation associated to Distributed Scatterers (DS) on the ground. More specifically, in this work, the SBAS technique (Berardino et al., 2002; Lanari et al., 2004) is used to retrieve the deformation, due to natural or anthropogenic causes, in some urban areas in Italy, at complementary scales of resolutions. As a matter of fact, the SBAS technique was initially developed to analyze sequences of multi-looked (averaged) interferograms (Berardino et al., 2002), but, subsequently, an adaptation of the SBAS algorithm that works at full spatial resolution scale (Lanari et al., 2004; Bonano et al., 2012) was proposed for the monitoring of buildings and public infrastructures, especially in urban areas. In this work, both approaches were used to retrieve mean deformation velocity maps as well as deformation time series related to PS and DS targets locations. Several papers describe the main operational steps of the SBAS methods, for instance, see Lanari et al. (2007).

Here, we summarize the basics of the method and the relevant processing stages. The starting point of SBAS is the generation of a sequence of SB DInSAR interferograms, which are characterized by small temporal and perpendicular baseline separations. First, the interferograms are unwrapped. 
Unwrapping operation (Costantini et al., 1998) permits to recover the full phase, i.e., not restricted to the $[-\pi, \pi]$ interval, from the measured wrapped phases. To this end, the space-time phase unwrapping technique, known as Extended Minimum Cost Flow (EMCF) approach (Pepe and Lanari, 2006), is usually applied to efficiently perform the unwrapping of the identified sequence of multi-temporal SB DInSAR interferograms. Subsequently, on a pixel-by-pixel basis, the unwrapped SB interferograms are inverted using Singular Value Decomposition (SVD) method (Strang, 1988) and a solution with a sound, physical meaning is found. Then, the constraint on the minimum velocity of the searched deformation time series is applied. Finally, atmospheric artifacts, as well as topographical phase residual components, are estimated and filtered out from the obtained deformation time series (Ferretti et al., 2001; Berardino et al., 2002). Figure 1(a) shows the simplified block diagram of the SBAS processing approach. In some cases, as said, the interest can be in studying the deformation of targets that are pointwise (i.e., not distributed over several pixels of the imaged scene). In the case the deformation of pointwise structures at the full spatial scale have to be studied, the extension of the SBAS technique (Lanari et al., 2004; Bonano et al., 2012) is adopted. The analysis starts with the modulo- $2 \pi$ subtraction, for each interferogram, of the Low-Pass (LP) phase components from the single-look interferograms. The residual phases are then inverted to get an estimate of the deformation signals related to pointwise structures and discriminate the actual signals from other residual phase artifacts, such as the topography of the area at full spatial scale as well as those related to Doppler Centroid (DC) frequency difference of the SB DInSAR pairs. Figure 1(b) shows the diagram block of the full-scale SBAS technique.

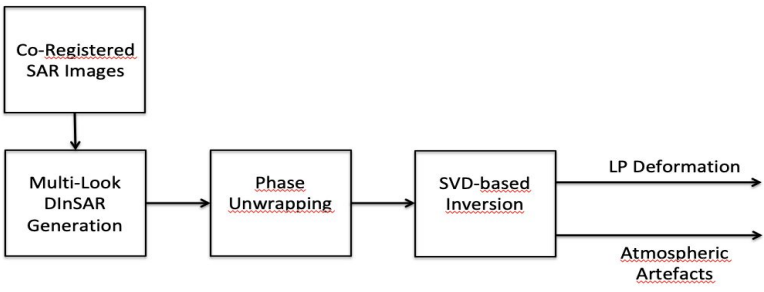

(a)

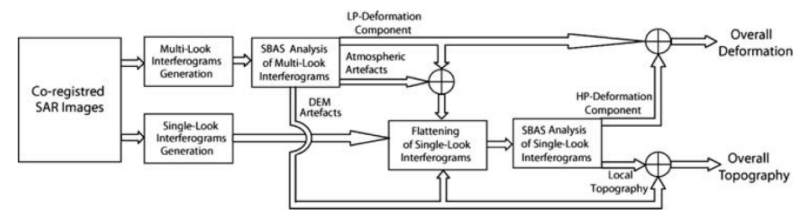

(b)

Figure 1. Processing chain of the SBAS algorithm at low- (a) and full- (b) spatial resolution scale, partly reproduced from Guzzetti et al. (2009)

SBAS algorithm is applied to the sequences of archived SAR images collected by the ERS and ENVISAT satellites of the European Space Agency (ESA) from 1992 to 2010. A maximum perpendicular baseline of $400 \mathrm{~m}$ and a maximum time span of two years have been used for the selection of the SB interferometric data pairs for the selected AOIs of Naples, Milan, and Turin in Italy. SBAS-DInSAR deformation data products are produced at a medium spatial scale and for some spotted areas in the residential and center areas of the selected AOIs also at the scale of every single building.

\section{TIME SERIES OF BIG GEODATA INTEROPERABLE SHARING}

New methods are needed to fill the gap between the form in which products derived by processing and analysis of EO data are provided by specific applications and the input needs of interoperable Web GISs so that the discovery and utilization of such products can improve. Moreover, depending on the expertise, scope, and roles of the users, different modalities have to be provided to ensure an effective discovery and utilization of BGD.

Within the URBAN GEO BIG DATA project, we consider as potential users of the Spatial Data Infrastructure (SDI) not only scientists but mainly public administrators of urban areas who may take advantage of the multisource and heterogeneous BGD for planning purposes. Besides, citizens are potentially interested in visualizing geodata of the urban areas where they live or plan to live related to mobility, three-dimensional buildings, ground deformation, soil consumption, land use, and land cover. This information impacts the quality of life as well as investment in real estates.

The preliminary method for enabling BGD utilization by such users is to provide them with a discovery facility to let them know what information is available through the SDI. To this end, scientists primarily need to know the semantics of the BGD, the accuracy, resolution and above all the process that generated the deformation time series in order to understand if the BGD are of interest to their studies. On the other hand, public administrators primarily need to use the data for planning purposes or may want to download and share them in the open data portals of their administrations. To this end, they need to know the temporal validity of the data, if data are subject to copyright, who is the contact person and the organization that owns the data, etc. Since public administrators are used to browsing metadata in Repertorio Nazionale dei Dati Territoriali (RNDT) format, defined as the standard for sharing geodata by Italian administrations, we have set up a catalog service of RNDT metadata to enable BGD querying by both free text keywords and faceted search.

The second method for enabling BGD utilization is to generate distinct products and services depending on the user roles. Deformation time series related to DS or PS targets on the ground, such as the ones generated by analyzing sequences of SB DInSAR interferograms, as explained in the previous section, although precise, are unsuitable for being easily understood by non-experts, who are a part of the target users of the project. Even for experts, we need to provide an easily understandable synthesis. In the following subsections, we describe the basic notions, methods, and processes aimed at the sharing of deformation time series derived through DInSAR measurements in urban areas, so that visualizations suitable for both experts and non-experts can be realized.

\subsection{Basic Notions of Web Service Interoperability}

Standard interoperable Web sharing of geodata can be conceived at two distinct levels. Firstly, at the semantic level, using the standard metadata formats, such as INSPIRE and its Italian extension RNDT, enriched with thesauri and ontologies, have been proposed to enable the discovery of geodata (Fugazza et al., 2014). As far as time series of geodata values (i.e., sequence of data values ordered in time with a common georeference) is concerned, an OGC standard exchange data 
format has been defined, the TimeseriesML (TSML), that is an XML encoding implementing the OGC Timeseries Profile of Observations and Measurements with the intent of allowing the exchange of such datasets across information systems. Through the use of OGC standards, TSML aims at being an interoperable exchange format that may be reused to address a range of data exchange requirements.

Secondly, at the syntactic level, OGC standards have been defined and are nowadays widely available to enable the discovery, access, visualization, query, and processing of geodata by defining an interface for Machine to Machine (MtM) communication. These OGC standards include Catalogue Service for the Web (CSW), WMS, Web Map Tile Service (WMTS), Web Feature Service (WFS), and Web Processing Service (WPS). Moreover, WMS with time support (WMS-T) is available, which is not an OGC standard but OGC suggests a best practice to serve time-dependent data, and map servers implement WMS-T conforming to the OGC best practice.

In the following, we describe the technologies adopted for interoperable sharing of DInSAR measurements targeting experts and non-experts.

\subsection{DInSAR Measurements Sharing}

DInSAR measurements were generated with a textual header encoding some metadata describing measurements' characteristics, such as temporal and spatial resolution and range, deformation unit, and geographic reference system. Besides, the pointwise measurements (temporal interferometric coherence, deformation velocity, and geographic coordinates, i.e., latitude and longitude) are reported together with the list of residual topography measurements. Such information constitutes a set of time series as conceived by TimeseriesML, one for each measurement point (latitude and longitude). What we need is to serve such a set of time series in a form suitable for two kinds of users: experts and non-experts. Since experts may need to analyze the quantitative data values varying in time by querying each single measurement point on a map, we have set up a WFS on DInSAR measurements. Moreover, a synthesis is provided by generating a map of the average deformation velocity of each point for the whole time span by enabling a WMS (see Figure 2).

To this end, the GET-IT tool (Lanucara et al., 2017) developed at CNR IREA within the flagship project RITMARE has been used provided that the DInSAR measurements data format has been parsed and translated as follows (see Figure 3):

- a CSV file that contains the average of the deformation velocities in order to create a WMS;

- a CSV file that contains the set of time series values, which is needed for configuring the WFS in order to retrieve the series of values related to any single measurement point;

- a CSV file, used for the WMTS service that contains in each row every single deformation value and its spatial and temporal coordinates;

- a simple GeoJSON file that contains a few metadata fields extracted from the original file used to fill the RNDT metadata;

- an XML file that contains the style to visualize the map (the color of the points visualized on the WMS map based on the average value and the size of the points based on the zoom level).
As far as non-experts are involved, information on deformation in time can be better perceived by providing a temporal series of images in which the values of each pixel are represented by distinct colors which reflect the accumulation of the deformation from the beginning of the time span. Such information is suitable for animated visualizations, as it is described in the next section, in order to be able to perceive at a glance the variation of the cumulative deformation from the beginning to the end of the time span

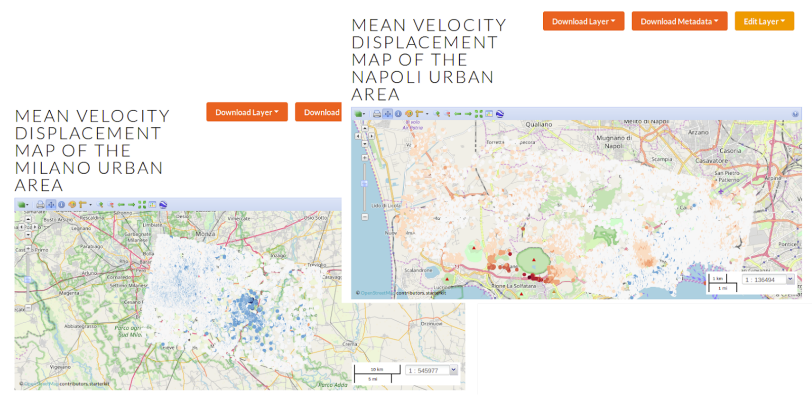

Figure 2. Mean deformation velocity maps of Milan and Naples deployed in the IREA data node, accessed via WMS

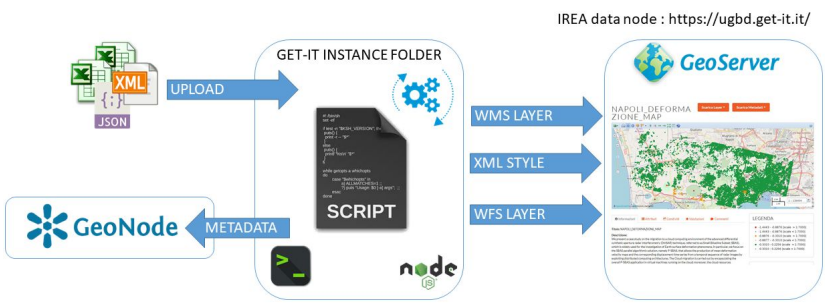

Figure 3. Web deployment of metadata, mean deformation velocity maps, and time series of cumulative deformation

In order to process the set of time series for generating both a raster layer for a single time and enabling WMS-T which compiles all the time-dependent raster layers, where all of them are tiled and served using WMTS, a parametric and automatic procedure has been designed and developed in Java. Besides the set of time series, the procedure takes as input the desired spatial resolution of the pixel (e.g., $80 \mathrm{~m}$ ), timestamp (e.g., year), and the thematic resolution (accuracy of deformation values, e.g., $5 \mathrm{~mm}$ ) and generates the following:

- a GeoJSON file for each interval of values for a single color in a year, provided that the minimum and maximum values of the distribution of values have been computed to quantify the data range appropriately;

- a GeoTIFF file for each interval of values for a single color in a year, created using the gdal_rasterize function of Geospatial Data Abstraction Library (GDAL) within Node.js code using as input each GeoJSON file created previously;

- a GeoTIFF file for each timestamp (e.g., year), created by merging the previous GeoTIFF files. This is performed using the gdal merge function of GDAL inside Node.js code. The GeoTIFF images are then served as WMS-T using the ImageMosaic plugin of GeoServer. Then, each GeoTIFF file is tiled using GeoWebCache integrated into GeoServer and served using WMTS (see Figure 4). 


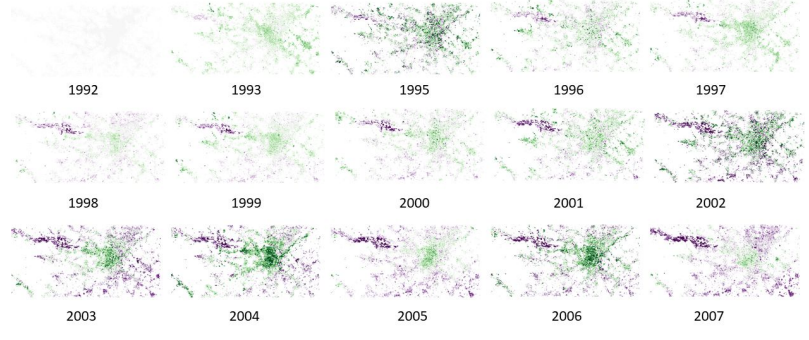

Figure 4. Raster time series of Turin

5.

\section{VISUALIZING DINSAR DEFORMATION TIME} SERIES WITH CESIUMJS

Visualization of DInSAR deformation time series is achieved using mainly CesiumJS and OGC standards through GeoServer. CesiumJS is a free and open source library that offers high-performance and cross-platform visualization and exploration of three-dimensional geographic data on the Web. All the libraries we use for visualization are Free and Open Source Software (FOSS). Moreover, we used VR-TheWorld Server to construct the terrain of the virtual globe built using CesiumJS. It provides a Digital Elevation Model (DEM) with a 90-meter resolution for the entire globe, including bathymetry. In the Web GIS, we provide six basemaps, which are Bing Maps Aerial, Mapbox Satellite Streets, OpenStreetMap, CARTO Dark, Stamen Terrain, and Stamen Watercolor. The ability to change the basemap enables the users to pick a basemap most suitable to see the overlaying colors. The Web GIS enables to switch between these six basemaps and the three cities. For each city either mean deformation velocity map or cumulative deformation animation can be turned on (see Figure $5)$.

The mean deformation velocity layers are retrieved from GeoServer using WMS and overlaid on the virtual globe. A single style is used for all the layers to enable comparison between different cities. The style is defined using OGC standard Styled Layer Descriptor (SLD) in GeoServer. Each deformation point can be clicked on to display its cumulative deformation time series using a plot (see Figure 5). The data used to create the plot is retrieved by making a WFS request to GeoServer.

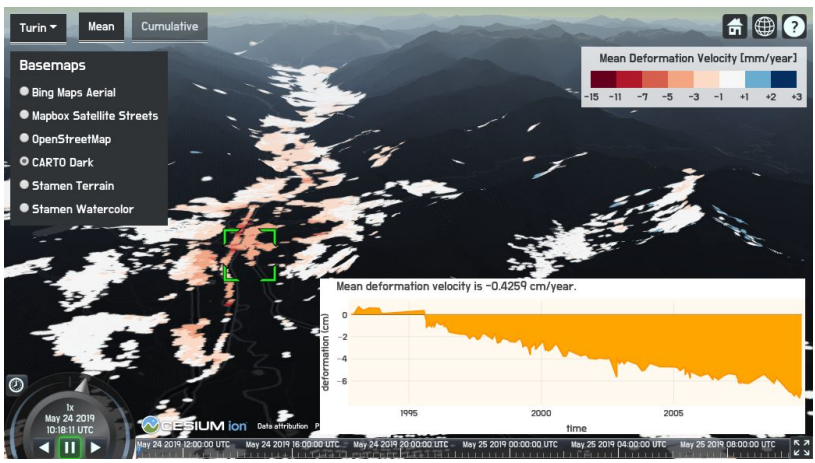

Figure 5. Mean deformation velocity visualization and query

The cumulative deformation raster layers, generated for each year from 1992 to 2010, are served using WMS-T, employing the ImageMosaic plugin of GeoServer. Each raster layer is then tiled using GeoWebCache integrated into GeoServer and served using WMTS. Then, we animate the raster layers using the animation and timeline widgets of CesiumJS to demonstrate the ground deformation of Naples, Milan, and Turin for 18 years at a glance using animation. Tiling the layers enhances the user experience during the animation.

The intervals for colors do not exceed the standard deviation of about $1 \mathrm{~mm} /$ year for mean deformation velocity and $5 \mathrm{~mm}$ for deformation (Casu et al., 2006). All the points having a value in an interval are colored using a single color. Moreover, as data have both negative and positive values, the color scheme used for visualization should adhere to diverging schemes where equal emphasis is put on mid-range critical values and extremes at both ends of the data range. In diverging schemes, the break in the middle is emphasized with light colors, and low and high extremes are emphasized with dark colors that have contrasting hues (Brewer, 1994). We chose the color scheme for diverging data with eleven classes using ColorBrewer 2.0 (Harrower et al., 2003). In this case, for both parts of the data range (negative and positive), the number of intervals do not exceed the suggested number of classes for choropleth maps, which is between five and seven. Considering the standard deviation, the fact that the nature of the data is diverging, and the suggested number of classes for choropleth maps, we use the color schemes visible in Figure 5 and 6 . In the color scheme of mean deformation velocity, the white color is for the mean deformation velocity values around zero, $[-1,1] \mathrm{mm} / \mathrm{year}$; the red hue is for the values less than $-1 \mathrm{~mm} /$ year; the blue hue is for the values higher than $1 \mathrm{~mm} / \mathrm{year}$. In the color scheme of cumulative deformation, the white color is for the deformation values around zero, $[-5,5] \mathrm{mm}$; the purple hue is for the values less than $-5 \mathrm{~mm}$; the green hue is for the values higher than $5 \mathrm{~mm}$. Also for cumulative deformation, the same style (color scheme) is used for all the cities to enable comparison among them. As the values for both mean deformation velocity and cumulative deformation concentrate around zero, the intervals become wider towards the low and high extremes.

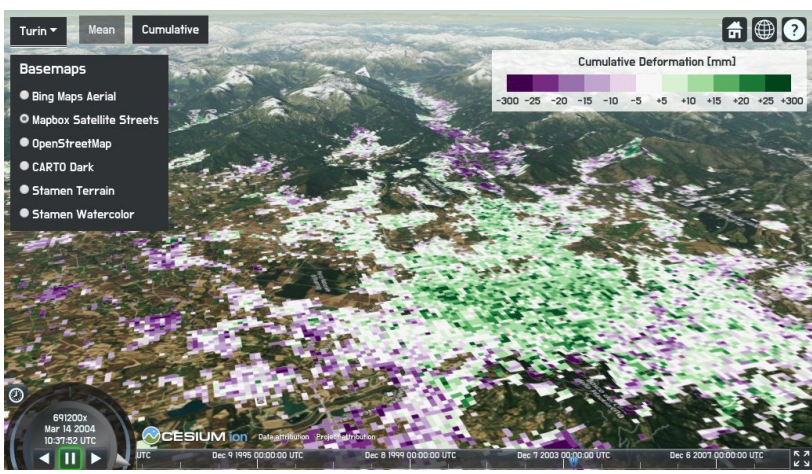

Figure 6. A raster layer of cumulative deformation animation

The animation can be initiated by pressing the play button in the animation widget. The speed of the animation can be adjusted using the same widget. Moreover, using the timeline widget, it is possible to scroll in time manually. The source code of the visualization can be found on GitHub ${ }^{1}$.

\section{CONCLUSIONS}

The primary concern when creating an SDI to support urban planning is to enable public administrators to discover, i.e., to query and retrieve BGD matching their needs. Thus we need to ensure that BGD semantics, formats, lineage, and ancillary information are adequately described by metadata and

\footnotetext{
${ }^{1}$ https://github.com/kilsedar/urban-geo-big-data-3d
} 
effectively indexed by catalog services. Moreover, to enable full exploitation of BGD, we need to provide access to it through interoperable standards and effective visualization.

In this paper, an experiment of generation, synthesis, sharing, and visualization of DInSAR deformation time series for three urban areas (i.e., Naples, Milan, and Turin) carried out within the URBAN GEO BIG DATA project is described. Besides the original algorithm for generating the time series, a novel methodology for synthesizing and sharing them is proposed. Synthesizing BGD is a preliminary and essential step for effectively sharing it. It aims at highlighting the most important characteristics and patterns within BGD, thus avoiding to overload the consumers with a massive amount of information by allowing them to focus only on the relevant aspects. We presented the implementation details of the method to synthesize and share DInSAR time series carried out using FOSS and OGC standards. The approach is scalable since the granulation of the measurements can be tuned by taking into account distinct aspects: the accuracy of the measurements so as not to highlight noise; the specific resources of the implementation platform that can impose a limit on the tile dimension; finally, the desired level of detail of the measurements that are needed by the targeted stakeholders. We believe that the proposed parametric approach is flexible enough to model situations characterized by distinct measurement accuracy, hardware characteristics, and user needs.

Visualization of the synthesis enable scientists, public administrators, and the citizens of urban areas detect spatio-temporal patterns of deformation. Using a virtual globe, thus incorporating terrain data enriches the visualization. The use of open standards and FOSS for Web maximizes interoperability, replicability, and reusability. In the future, the same methodology will also be used for Padua and Rome, which are the other two cities that are in the focus of URBAN GEO BIG DATA project.

\section{ACKNOWLEDGMENTS}

This research is funded by the project URBAN GEO BIG DATA, a project of national interest (PRIN), funded by the Italian Ministry of Education, University and Research (MIUR)—id 20159CNLW8.

\section{REFERENCES}

Andrienko, N., Andrienko, G., 2006. Exploratory Analysis of Spatial and Temporal Data: a Systematic Approach. Springer Science \& Business Media.

Berardino, P., Fornaro, G., Lanari R., Sansosti E., 2002. A new algorithm for surface deformation monitoring based on small baseline differential SAR interferograms. IEEE Transactions on Geoscience and Remote Sensing 40(11), pp. 2375-2383.

Bonano, M., Manunta, M., Marsella, M., Lanari, R., 2012. Long term ERS/ENVISAT deformation time-series generation at full spatial resolution via the extended SBAS technique. International Journal of Remote Sensing 33(15), pp. 4756-4783. doi.org/10.1080/01431161.2011.638340.

Brewer, C. A., 1994. Color Use Guidelines for Mapping and Visualization. Visualization in Modern Cartography, pp. 123-148.
Casu, F., Manzo, M., Lanari, R., 2006. A quantitative assessment of the SBAS algorithm performance for surface deformation retrieval from DInSAR data. Remote Sensing of Environment 102(3-4), pp. 195-210.

Costantini, M., 1998. A Novel phase unwrapping method based on network programming. IEEE Transactions on Geoscience and Remote Sensing 36(3), pp. 813-821.

Ferretti, A., Prati, C., Rocca, F., 2001. Permanent scatterers in SAR interferometry. IEEE Transactions on Geoscience and Remote Sensing 39(1), pp. 8-20.

Fugazza, C., Pepe, M., Oggioni, A., Pavesi, F., Carrara, P., 2014. A Holistic, Semantics-aware Approach to Spatial Data Infrastructures. In: Proceedings of 3rd International Conference on Data Management Technologies and Applications, pp. 349-356. doi.org/10.5220/0004997603490356.

Guzzetti, F., Manunta, M., Ardizzone, F., Pepe, A., Cardinali, M., Zeni, G., Reichnbach, P., Lanari, R., 2009. Analysis of Ground Deformation Detected Using the SBAS-DInSAR Technique in Umbria, Central Italy. Pure and Applied Geophysics 166(8), pp. 1425-1459.

Harrower, M., Brewer, C. A, 2003. ColorBrewer.org: An Online Tool for Selecting Colour Schemes for Maps. The Cartographic Journal 40(1), pp. 27-37.

Harrower, M., Fabrikant, S., 2008. The role of map animation for geographic visualization. Geographic Visualization: Concepts, Tools and Applications, pp. 44-66.

Imperatore, P., Pepe, A., 2016: Geospatial Technology: Environmental and Social Applications, IntechOpen.

Kampes, B., 2006. Radar Interferometry: Persistent Scatterer Technique. The Netherlands: Springer.

Lanari, R., Casu, F., Manzo, M., Zeni, G., Berardino, P., Manunta, M., Pepe, A., 2007. An Overview of the Small BAseline Subset Algorithm: a DInSAR Technique for Surface Deformation Analysis. Pure and Applied Geophysics 164(4), pp. 637-661.

Lanari, R., Mora, O., Manunta, M., Mallorqui, J. J., Berardino, P., Sansosti, E., 2004. A Small baseline approach for investigating deformation on full resolution differential SAR interferograms. IEEE Transactions on Geoscience and Remote Sensing 42(7), pp. 1377-1386.

Lanucara, S., Oggioni, A., Modica, G., Carrara, P., 2017. Interoperable Sharing and Visualization of Geological Data and Instruments: A Proof of Concept. In: International Conference on Computational Science and Its Applications, pp. 584-599.

MacEachren, A. M., Kraak, M. J., 2001. Research Challenges in Geovisualization. Cartography and Geographic Information Science 28(1), pp. 3-12.

Massonet, D., Feigl, K. L., 1998. Radar interferometry and its application to changes in the Earth's surface. Reviews of Geophysics 36(4), pp. 441-500.

Mora, O., Mallorqui, J. J., Broquetas, A., 2003. Linear and nonlinear terrain deformation maps from a reduced set of 
interferometric SAR images. IEEE Transactions on Geoscience and Remote Sensing 41(10), pp. 2243-2253.

Ogao, P. J., Kraak, M. J., 2002. Defining visualization operations for temporal cartographic animation design. International Journal of Applied Earth Observation and Geoinformation 4(1), pp. 23-31.

Peltzer, G., Rosen, P. A., 1995. Surface Displacement of the 17 May 1993 Eureka Valley, California, Earthquake Observed by SAR Interferometry. Science 268(5215), pp. 1333-1336.

Pepe, A., Bonano, M., Bordogna, G., Brovelli, M., Calò, F., Carrara, P., Congedo, L, Frigerio, L., Imperatore, P., Lanari, R., Lanucara, S., Manzo, M., Munafò, M., 2018. The "Urban Geomatics for Bulk Information Generation, Data Assessment and Technology Awareness" Project: Detection, Representation and Analysis of the Urban Scenario Changes. In: International Geoscience and Remote Sensing Symposium (IGARSS), pp. 2902-2905.

Pepe, A., Lanari, R., 2006. On the Extension of the Minimum Cost Flow Algorithm for Phase Unwrapping of Multitemporal Differential SAR Interferograms. IEEE Transactions on Geoscience and Remote Sensing 44(9), pp. 2374-2383.

Peuquet, D. J., 1994. It's About Time: A Conceptual Framework for the Representation of Temporal Dynamics in Geographic Information Systems. Annals of the Association of American Geographers 84(3) pp. 441-461.

Shneiderman, B., 1996. The Eyes Have It: A Task by Data Type Taxonomy for Information Visualizations. In: Proceedings of the IEEE Symposium on Visual Languages, pp. 336-343.

Strang, G., 1988: Linear Algebra and Its Applications, Thomson Learning. 